Pacific

Journal of

Mathematics

CONTRACTION CRITERIA FOR REDUCIBLE RATIONAL CURVES WITH COMPONENTS OF LENGTH ONE IN SMOOTH COMPLEX THREEFOLDS

TOM ZERGER 


\title{
CONTRACTION CRITERIA FOR REDUCIBLE RATIONAL CURVES WITH COMPONENTS OF LENGTH ONE IN SMOOTH COMPLEX THREEFOLDS
}

\author{
TOM ZERGER
}

\begin{abstract}
Let $X$ be a smooth complex threefold and $C$ a linear chain of $\boldsymbol{n}$ smooth rational curves in $X$, each intersecting the canonical sheaf $\mathcal{K}_{X}$ trivially, and each having length 1 , where the length is Kollár's invariant. Formal criteria will be given to determine when $C$ contracts, when $C$ deforms, and when $C$ neither contracts or deforms in $\hat{X}$, the formal completion of $X$. It is shown precisely, using the curve $C$, its components, and their defining ideals, how the behavior of $C$ coincides with the deformation theory of the compound $A_{n}$ singularity.
\end{abstract}

\section{Introduction.}

Let $C=\bigcup_{i=1}^{n} C_{i}$ be a linear chain of $n$ smooth rational curves $C_{i}$ of length 1 in a smooth complex threefold $X$. Furthermore, assume $\mathcal{K}_{X} \cdot C_{i}=0$ for each $i$ and that each $C_{i}$ has a rational formal neighborhood in $X$.

The main purpose of this paper is to describe explicitly the deformations of $C$ in $\hat{X}$, the formal completion of $X$. Formal criteria will be given to determine when $C$ contracts formally and when $C$ deforms formally in $\hat{X}$. Before contraction criteria can be stated it is important to make clear what is meant by a formal contraction and a formal deformation. In particular, the definitions of formal cDV modification and formal cDV contraction will be made precise. These definitions are motivated by the deformations of DuVal singularities and by the formal constructions that were utilized by Reid [14] and Jiménez [6] in determining contraction criteria for a single smooth rational curve; Reid considered length 1 curves and Jiménez curves of length greater than 1.

Assume $f: X \rightarrow Y$ of $C=\bigcup_{i=1}^{n} C_{i}$ is an analytic contraction with $f(C)=q$ so that $f: X \backslash C \rightarrow Y \backslash q$ is an isomorphism. Let $m_{q, Y}$ be the maximal ideal at the point $q \in Y$. 
Definition 1.1 ([3, Kollár, p. 95]). Let $f: X \rightarrow Y$ contract $C$ to the point $q \in Y$. The length of the component $C_{i}$ is the length of the scheme with structure sheaf $\mathcal{O}_{X} / f^{-1}\left(m_{q, Y}\right)$ at a generic point of $C_{i}$.

Now $C$ is a closed subscheme of $X$ and $q$ is a closed subscheme of $Y$, and, therefore, $\hat{X}$ is supported on $C$ and $\hat{Y}$ is supported on $q$. Reid [14] has shown that $q$ is a compound DuVal (cDV) singularity, that is, a general hyperplane section $Y_{0}$ of $q$ has a DuVal singularity at $q$. Therefore, $q$ is a singularity of type $c A_{n}(n \geq 1), c D_{n}(n \geq 4), c E_{6}, c E_{7}$, or $c E_{8}$. Furthermore, the induced map on surfaces $X_{0}=f^{*} Y_{0} \rightarrow Y_{0}$ is a factor of the minimal resolution of $q \in Y_{0}$.

Definition 1.2. A formal cDV modification consists of a map $\hat{f}: \hat{X} \rightarrow$ $\hat{Y}$ of formal threefolds, with $\hat{X}$ supported on a curve $C$ and $\hat{Y}$ supported on a point $q$, such that a general section $s \in m_{q}$, the maximal ideal at $q$, defines a formal DuVal surface singularity, while $\hat{f}^{-1}(s)$ defines a formal partial resolution.

The formal length of $C_{i}$ is analogous to Definition 1.1 with $\hat{f}$ a formal $\mathrm{cDV}$ modification. Certainly what is preferred is that a formal cDV modification is equivalent to a formal modification as defined by Artin [2, Defn. 1.7], because Artin shows in [2, Thrm. 3.1] and [1, Thrm. 6.2] that the existence of a formal modification implies $C$ contracts or deforms in the analytic category. At the end of this section, after more evidence is acquired, it will be conjectured that these two definitions are equivalent, at least for the curve considered in this paper.

From the definition of a formal cDV modification and the semi-universal property of deformations of DuVal singularities and their simultaneous partial resolutions, a formal cDV contraction can be defined. This theory has been described by Tyurina [15], Kas [7], Pinkham [13], Reid [14], as well as Katz and Morrison [8]. All of the works cited are in the analytic category, so for ease in referring to these, the general brief discussion following and the defining equations given explicitly in Section 2.2 for the $A_{n}$ singularity, which mainly follows the notation in [8], will be in the analytic category. However, by taking formal completions of these spaces, analogous results are obtained in the formal category for formal DuVal singularities. When necessary for clarity, these formal completions will be described in more detail.

Let $(\pi, \mathcal{Y}, \Sigma)$ and $(\sigma, \mathcal{X}, T)$ be the semi-universal families of the deformations of $Y_{0}$ and the partial resolution $X_{0}$, respectively. It is known, see [15], that from the defining equation for the singular space $Y_{0}$ the threefold $Y$ can be viewed as the total space of a one-parameter family $\left\{Y_{t}\right\}$. Therefore, there is a map $h: \Delta_{t} \rightarrow \Sigma$ that classifies $Y$. The space $X$, then, can be viewed as the corresponding one-parameter family $\left\{X_{t}\right\}$ described by $g: \Delta_{t} \rightarrow T$ in the diagram below. 


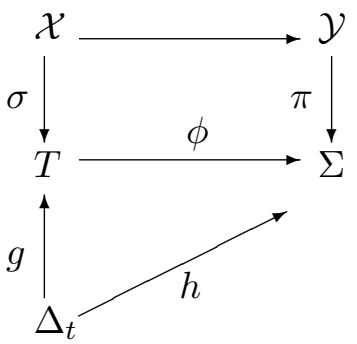

Figure 1.

More precisely, $X=g^{*}(\mathcal{X})$ and $Y=h^{*}(\mathcal{Y})$. The curve $\mathcal{C}$ is the exceptional set in $\mathcal{X}$ that lies over the discriminant in the base space $T$. Determining if $C$ contracts or components of $C$ deform is accomplished by looking at the discriminant locus of $T$ and observing when $g$ factors through.

If $\hat{f}: \hat{X} \rightarrow \hat{Y}$ is a formal cDV modification, then $\{s=t\}$ gives a oneparameter family of hypersurfaces defining a formal deformation of the singular space, $\hat{Y}_{0}$, given by $s=0$. Therefore, the inverse image of this family under $\hat{f}$ is a formal partial resolution of singularities. The semi-universal property of deformations of DuVal singularities and their partial resolutions shows that $\hat{f}: \hat{X} \rightarrow \hat{Y}$ is isomorphic to the induced map $g^{*}(\hat{\mathcal{X}}) \rightarrow h^{*}(\hat{\mathcal{Y}})$, where $\hat{\mathcal{X}}$ and $\hat{\mathcal{Y}}$ are the semi-universal families of the formal deformation of $\hat{X}_{0}$ and $\hat{Y}_{0}$, respectively. Taking formal completions of the spaces $\Delta_{t}$ and $T$ we have the following situation:

Definition 1.3. $\hat{f}: \hat{X} \rightarrow \hat{Y}$ is a formal cDV contraction if $\hat{f}: \hat{X} \rightarrow \hat{Y}$ is a formal $\mathrm{cDV}$ modification such that the general section $s \in m_{q}$ defines a map $\hat{g}: \hat{\Delta}_{t} \rightarrow \hat{T}$ which does not factor through the inclusion of the discriminant locus in $\hat{T}$.

With these definitions of formal cDV modification and formal cDV contraction, formal results have been established that are analogous to Artin's results in the analytic case.

Proposition 1.4. If $\hat{f}: \hat{X} \rightarrow \hat{Y}$ is a formal cDV modification, then $\hat{f}$ is either a formal cDV contraction, or some component of $C$ has a formal deformation.

Proof. As $C$ lies over the discriminant locus, the components of $C$ that deform can be determined from its locally closed subsets. Over each subset is a flat family of deformations of some corresponding subset of $C$. So, if $\hat{g}$ factors through, then pulling back the flat family to $\hat{\mathcal{X}}$ is the formal deformation of this component of $C$. 
If $\hat{g}$ does not factor through the discriminant locus, then by definition $\hat{f}$ is a formal $\mathrm{cDV}$ contraction.

Conjecture 1.5. If $\hat{f}: \hat{X} \rightarrow \hat{Y}$ is a formal cDV modification, then $\hat{f}$ is a formal modification in the sense of Artin [2, Defn. 1.7].

With these definitions established, contraction criteria can be established. To know which cDV deformation space to utilize it must first be known which cDV singularity results if $C$ contracts. This analytic result is immediate from the construction of the formal $c A_{n}$ modification $\hat{f}$ in Section 3, which utilizes the conormal sheaf on $C$ and its restrictions to the components $C_{i}$.

Theorem 1.6. If $f: X \rightarrow Y$ is an analytic contraction map with $f(C)=q$ and $C=\bigcup_{i=1}^{n} C_{i}$ with all components having length 1 , then a general hyperplane section of $q$ has an $A_{n}$ type singularity at $q$.

Section 4, then, explicitly determines when $C$ deforms formally in $\hat{X}$ and when a formal cDV contraction exists. More precisely, a method similar to Reid's "Pagoda" construction in [14] shows that the contractibility of $C$ can be detected in its higher order neighborhoods. The construction results in a sequence of defining ideals

$$
\mathcal{K}_{m} \subset \mathcal{K}_{m-1} \subset \cdots \subset \mathcal{K}_{2} \subset \mathcal{K}_{1}=\mathcal{I}
$$

where $\mathcal{I}$ is the reduced ideal sheaf of $C$ in $X$ and

$$
0 \longrightarrow \mathcal{O}_{C} \longrightarrow \mathcal{K}_{i} / \mathcal{I} \mathcal{K}_{i} \longrightarrow \omega_{C}^{*} \longrightarrow 0
$$

is exact for each $m \leq i \leq 1$ with $\omega_{C}^{*}$ the dual of the dualizing sheaf on $C$. This sequence of ideals can be extended if and only if the the exact sequence splits. Comparing the construction of this sequence of ideals defining $C$ with the semi-universal deformation of the $A_{n}$ singularity at $q$, the following two theorems are proved:

Theorem 1.7. $C$ deforms formally in $X$ if and only if there exists an infinite chain of subsheaves $\cdots \subset \mathcal{K}_{m+1} \subset \mathcal{K}_{m} \subset \cdots \subset \mathcal{K}_{2} \subset \mathcal{I}$ such that $\mathcal{K}_{m} / \mathcal{K}_{m+1} \cong \mathcal{O}_{C}$ and $\mathcal{K}_{m+1} / \mathcal{I} \mathcal{K}_{m} \cong \omega_{C}^{*}$, where $\omega_{C}^{*}$ is the dual of the dualizing sheaf.

Theorem 1.8. A formal $c A_{n}$ contraction of $C$ exists if and only if there is no infinite chain of subsheaves $\cdots \subset \mathcal{K}_{m+1} \subset \mathcal{K}_{m} \subset \cdots \mathcal{K}_{2} \subset \mathcal{I}_{D}$ satisfying $\mathcal{K}_{m} / \mathcal{K}_{m+1} \cong \mathcal{O}_{D}$ and $\mathcal{K}_{m+1} / \mathcal{I} \mathcal{K}_{m} \cong \omega_{D}^{*}$ for any $D=\bigcup_{j=i}^{k} C_{j}(1 \leq i \leq k \leq$ $n)$, where $\mathcal{I}_{D}$ is the ideal sheaf of $D$ in $X$ and $\omega_{D}^{*}$ is the dual of the dualizing sheaf.

Some immediate consequences of these theorems and the construction involved is that not all chains of $(1,1)$ curves will contract, though each 
component could be contracted separately (see Example 4.13). Also, unlike the single component case, it is not true that a chain of length 1 curves will either contract or deform.

\section{Preliminaries: Length and equations for deformations of $A_{n}$ singularities.}

In Section 2.1, we briefly discuss the significant role that the length of the components of $C$ plays in determining the higher order neighborhoods of $C$. Section 2.2 gives a more precise discussion of the semi-universal deformations of $A_{n}$ singularities and their simultaneous resolutions than in Section 1. See Figure 1 in Section 1 in reference to the maps and spaces discussed. Again, the notation utilized here is most similar to that in [8].

2.1. Length. Let $\mathcal{I}_{i} / \mathcal{I}_{i}{ }^{2}$ be the conormal sheaf of $C_{i}$ in $X$, which is locally free of rank two, so $\mathcal{I}_{i} / \mathcal{I}_{i}{ }^{2} \cong \mathcal{O}_{C_{i}}(a) \oplus \mathcal{O}_{C_{i}}(b)$ for integers $a$ and $b$. It will be written that $C_{i}$ is a curve of type $(a, b)$. The assumption that $\mathcal{K}_{X} \cdot C_{i}=0$ implies, by adjunction, that $a+b=2$, and the assumption that $C$ has a rational formal neighborhood in $X, H^{1}\left(\hat{X}, \mathcal{O}_{\hat{X}}\right)=0$, implies each $C_{i}$ is a curve of type $(1,1),(0,2)$ or $(-1,3)$. It is known, see [3, page 95$]$, that since each component has length 1 , they are all curves of type $(1,1)$ or $(0,2)$. As in the work of Jiménez in [6], it is because of the existence of a projection to $\mathcal{O}(-1)$ that the $(-1,3)$ curve has length greater than 1 .

As the methods in this paper are for a curve $C$ with multiple components, it is necessary to confirm that the existence of $\mathcal{O}_{i}(-1)$ factors has a similar effect on the length of each component curve $C_{i}$. Assume there is a finite sequence of defining ideals $\mathcal{I}=\mathcal{J}_{1} \supset \cdots \supset \mathcal{J}_{k}$ with $\mathcal{J}_{l} / \mathcal{J}_{l+1} \cong \mathcal{O}_{m(l)}(-1)$ for all $1 \leq l \leq k-1$ and some $m(l) \in\{1,2, \ldots, n\}$, and assume that the sequence cannot be extended to $\mathcal{J}_{k+1}$. If $\mathcal{J}_{k} / \mathcal{J}_{k}^{2}$ is generated by global sections and these can be lifted to global sections of $\hat{\mathcal{J}}_{k}$, then, as in [6], they define a formal cDV modification $\hat{f}: \hat{X} \rightarrow \hat{Y}$ with $\hat{f}^{-1}\left(m_{q}\right)=\hat{\mathcal{J}}_{k}$. The significance of all quotients being $\mathcal{O}_{m(l)}(-1)$ for some $m(l)$ is that $H^{0}\left(\mathcal{J}_{k}\right)=$ $H^{0}(\mathcal{I})$. By definition, the formal length of a component $C_{i}$ is the length of $\mathcal{O}_{\hat{X}} / \hat{\mathcal{J}}_{k}$ at a generic point of $C_{i}$. Let $p \in C_{i}$ with $p \notin C_{j}$ for $j \neq i$ be a generic point. We have $\mathcal{J}_{l}=\mathcal{J}_{l+1}$ unless $\mathcal{J}_{l} / \mathcal{J}_{l+1} \cong \mathcal{O}_{i}(-1)$. In this case, then, there is a longest subsequence $\mathcal{I}=\mathcal{J}_{l_{1}} \supset \cdots \supset \mathcal{J}_{l_{j}}$ such that $\mathcal{J}_{l_{t}} / \mathcal{J}_{l_{t+1}} \cong \mathcal{O}_{i}(-1)$ for $1 \leq t \leq j-l$. The length of $C_{i}$ is the length of $\mathcal{O}_{X} / \mathcal{J}_{l_{j}}$, which is $l_{j}$. In conclusion, this means that if we have a sequence $\mathcal{I}=\mathcal{J}_{1} \supset \cdots \supset \mathcal{J}_{l}$ and there is an ideal $\mathcal{J}_{l+1} \subset \mathcal{J}_{l}$ such that $\mathcal{J}_{l} / \mathcal{J}_{l+1} \cong$ $\mathcal{O}_{i}(-1)$, then the formal length of the component $C_{i}$ increases by 1 .

2.2. Equations for deformations of $\mathbf{A}_{\mathbf{n}}$ singularities. We are interested in the case where $Y_{0}$ is a singular surface having an $A_{n}$ singularity. Near the singularity with coordinates $\{x, y, z\}, Y_{0}$ is the hypersurface in 
$\mathbf{C}^{\mathbf{3}}(x, y, z)$ defined by the equation $-x y+z^{n+1}$. The analytic space $\mathcal{Y}$ is defined as the hypersurface in $\mathbf{C}^{\mathbf{3}}(x, y, z) \times \mathbf{C}^{\mathbf{n}}\left(\sigma_{1}, \ldots, \sigma_{n}\right)$ defined by

$$
-x y+z^{n+1}+\sigma_{1} z^{n-1}+\cdots \sigma_{n-1} z+\sigma_{n}=0 .
$$

The base space is $\Sigma=\mathbf{C}^{\mathbf{n}}\left(\sigma_{1}, \ldots, \sigma_{n}\right)$ and the map $\pi: \mathcal{Y} \rightarrow \Sigma$ is the map induced by projection.

The resolution corresponding to the semi-universal family can also be explicitly described. Let $T$ be the hyperplane in $\mathbf{C}^{\mathbf{n}+\mathbf{1}}\left(t_{1}, \ldots, t_{n+1}\right)$ defined by $\sum_{i=1}^{n+1} t_{i}=0$. The map on the base spaces, $\phi: T \rightarrow \Sigma$, is defined by $\sigma_{i}=$ the $(i+1)$ st symmetric polynomial in the $t_{i}$. Notice that by definition $\sigma_{0}=\sum_{i=1}^{n+1} t_{i}=0$. The smooth deformation $\sigma: V \rightarrow T$ induced by $\phi$ is defined in $\mathbf{C}^{\mathbf{3}}(x, y, z) \times \mathbf{C}^{\mathbf{n}+\mathbf{1}}\left(t_{1}, \ldots, t_{n+1}\right)$ by the equations

$$
\sum_{i=1}^{n+1} t_{i}=0,-x y+\prod_{i=1}^{n+1}\left(z+t_{i}\right)=0 .
$$

Now, define a mapping $V \rightarrow\left(\mathbf{P}^{\mathbf{1}}\right)^{n}$ by

$$
\left(x, y, z, t_{1}, \ldots, t_{n+1}\right) \rightarrow\left\{x, \prod_{j=1}^{i}\left(z+t_{j}\right)\right\}_{i}
$$

for $i=1, \ldots, n$. The analytic space $\mathcal{X}$, then, is defined to be the closure of the graph of this map, and the mapping $\sigma: \mathcal{X} \rightarrow T$ is defined by projection. If $\left(u_{k}, v_{k}\right)$ are the homogeneous coordinates on the $k$ th $\mathbf{P}^{\mathbf{1}}$ from the resolution, then the equations defining $\mathcal{X}$ are

$$
\begin{gathered}
-x y+\prod_{i=1}^{n+1}\left(z+t_{i}\right)=0, \\
x v_{j}=u_{j} \prod_{i=1}^{j}\left(z+t_{i}\right) \quad(1 \leq j \leq n), \\
\prod_{i=k+1}^{j}\left(z+t_{i}\right) u_{j} v_{k}=u_{k} v_{j}(1 \leq k<j \leq n) .
\end{gathered}
$$

From these equations it has been shown that $C$ is the exceptional set of the fiber of $\mathcal{X}$ over $\left(t_{1}, t_{2}, \ldots, t_{n+1}\right)=\overrightarrow{0}$ and the component $C_{i}$ is defined by $x=y=z=0, u_{j}=0$ for $j<i$ and $v_{k}=0$ for $k>i$. Furthermore, the curve $C_{i}+C_{i+1}+\cdots+C_{j}$ deforms when $t_{i}=t_{j+1}$.

Since $X$ is being viewed as the space of a one-parameter family of deformations of a resolution of an $A_{n}$ singularity, $X$ is recovered, as described by Pinkham in [13], from $g: \Delta_{t} \rightarrow T$. The coordinates $t_{i}$ of $T$, then, can be expressed as functions of $t$ vanishing at $t=0$. Let $g_{i}(t)=t_{i}$ under this parameterization, where the $g_{i}$ are holomorphic functions on a neighborhood 
of $0 \in \mathbf{C}$. These functions have a power series expansion near $t=0$. Let

$$
g_{i}(t)=\sum_{j=1}^{\infty} a_{i j} t^{j} \quad(1 \leq i \leq n+1) .
$$

Note that for our situation, we can only assume that the $g_{i}$ are formal functions. Pulling back $\mathcal{X}$ via $g, X$ can now be described from the equations defining the resolution with coordinates $\left\{x, y, z,\left(u_{i}, v_{i}\right), t\right\}$. In particular, we will be interested in defining $X$ near an intersection point of two components, $C_{i}$ and $C_{i+1}$ with $1 \leq i \leq n-1$. Now $C_{i} \cong \mathbf{P}^{\mathbf{1}}\left(u_{i}, v_{i}\right), C_{i+1} \cong \mathbf{P}^{\mathbf{1}}\left(u_{i+1}, v_{i+1}\right)$ and $X$ is defined near this point of intersection by the transition functions on the coordinate patches $\left(u_{i-1}, v_{i}, t\right),\left(u_{i}, v_{i+1}, t\right)$ and $\left(u_{i+1}, v_{i+2}, t\right)$, with the intersection point being in the coordinate patch $\left(u_{i}, v_{i+1}, t\right)$. These transition functions are:

$$
\begin{aligned}
u_{i-1} & =u_{i}^{2} v_{i+1}+u_{i}\left(g_{i}(t)-g_{i+1}(t)\right) \\
v_{i} & =1 / u_{i} \\
t & =t \\
u_{i+1} & =1 / v_{i+1} \\
v_{i+2} & =v_{i+1}^{2} u_{i}+v_{i+1}\left(g_{i+2}(t)-g_{i+1}(t)\right) \\
t & =t
\end{aligned}
$$

with the convention that if $i=1$ then $u_{i-1}=x$, and if $i=n-1$ then $v_{i+2}=y$.

Since the deformation of $\cup_{i=j}^{k} C_{i}$ occurs when $t_{j}=t_{k+1}$, the deformation of this curve is determined by whether it coincides with the discriminant locus of $g$ in $T$, which is $g_{j}(t)=g_{k+1}(t)$. In particular, the whole curve $C$ deforms when $g_{1}(t)=g_{n+1}(t)$. By viewing these functions as formal functions, we have:

Theorem 2.1. $C=\bigcup_{i=1}^{n} C_{i}$ deforms formally in $\hat{X}$ if and only if $g_{1}(t)=$ $g_{n+1}(t)$.

Theorem 2.2. $C=\bigcup_{i=1}^{n} C_{i}$ can be contracted via a formal cDV contraction if and only if $g_{i}(t) \neq g_{j}(t)$ for any $1 \leq i<j \leq n+1$.

These results and the transition functions defining $X$, Equation (2), will be utilized in Section 4.

\section{The singularity q.}

As mentioned above, in this case it is necessarily assumed that $\mathcal{I}_{i} / \mathcal{I}_{i}{ }^{2}=(1,1)$ or $(0,2)$ for all $i$. We will identify the rational double point by investigating 
the general section of $\hat{\mathcal{I}}$, which is obtained by lifting it from the globally generated conormal sheaf, $\mathcal{I} / \mathcal{I}^{2}$, on $C$.

The exact sequence of sheaves obtained by restricting the conormal sheaf of $C$ to the component $C_{i}$,

$$
0 \longrightarrow \mathcal{I}_{i} \mathcal{I} / \mathcal{I}^{2} \longrightarrow \mathcal{I} / \mathcal{I}^{2} \longrightarrow \mathcal{I} / \mathcal{I}_{i} \mathcal{I} \longrightarrow 0
$$

and its long exact cohomology sequence allow us to determine this global information.

The defining ideal sheaves for the component curves can be defined in local coordinates $\{x, y, z\}$ at the point of intersection $p=(0,0,0)$ of $C_{i}$ and $C_{j}$ by $\mathcal{I}_{i}=(x, z), \mathcal{I}_{j}=(y, z)$ and $\mathcal{I}=(x y, z)$. Then $\mathcal{I} /\left.\mathcal{I}^{2}\right|_{C_{i}}=\mathcal{I} / \mathcal{I}_{i} \mathcal{I}$ is locally free of rank 2 on $C_{i}$, generated by $\{x y, z\}$ at a point of intersection, $\mathcal{I}_{i} / \mathcal{I}_{i}{ }^{2}$ and $\mathcal{I}_{j} / \mathcal{I}_{j}{ }^{2}$ are generated by $\{x, z\}$ and $\{y, z\}$, respectively, and $\mathcal{I}_{i} \mathcal{I} / \mathcal{I}^{2}$ is locally free of rank 2 on $\cup_{j \neq i} C_{j}$. The map on generators of the inclusion map $\mathcal{I} / \mathcal{I}_{i} \mathcal{I} \hookrightarrow \mathcal{I}_{i} / \mathcal{I}_{i}{ }^{2}$ at a point of intersection is given by $x y \mapsto y \cdot x, z \mapsto z$, with $y$ a local coordinate on $C_{i}$. The determinant of this map, then, vanishes to order one at each point of intersection. Since $\mathcal{I}_{i} / \mathcal{I}_{i}{ }^{2}$ has degree two, $\mathcal{I} / \mathcal{I}_{i} \mathcal{I}$ has degree 0 if there are two points of intersection and degree 1 if there is just one. We have,

$$
\mathcal{I} / \mathcal{I}_{i} \mathcal{I}= \begin{cases}(0,0),(-1,1), \text { or }(-2,2) & \text { if } \quad 2 \leq i \leq n-1 \\ (0,1) \text { or }(-1,2) & \text { if } i=1 \text { or } n\end{cases}
$$

But, since each component has a rational formal neighborhood there can be no $\mathcal{O}(-2)$ factors and since it is assumed that each component has length 1 , there can be no $\mathcal{O}(-1)$ factors, as discussed in Section 2.1. Therefore, it is necessary that $\mathcal{I} / \mathcal{I}_{i} \mathcal{I}=(0,0)$ for $2 \leq i \leq n-1$ and $(0,1)$ for $i=1, n$.

The exact sequence, from the normalization of $C$, is

$$
0 \longrightarrow \mathcal{I} / \mathcal{I}^{2} \longrightarrow \bigoplus_{i=1}^{n} \mathcal{I} /\left.\mathcal{I}^{2}\right|_{C_{i}} \longrightarrow \bigoplus_{p \in C_{i} \cap C_{j}} \mathcal{I} /\left.\mathcal{I}^{2}\right|_{p} \longrightarrow 0
$$

That is, the middle term in the sequence is $\pi_{*} \pi^{*} \mathcal{F}$, where $\pi: \widetilde{C} \rightarrow C$ is the normalization of $C$ such that $\widetilde{C}$ is the disjoint union of the smooth component curves. Therefore, the first map is an isomorphism away from the intersection points and the second map is defined by $\left(s_{i}\right) \mapsto \oplus_{p \in C_{i} \cap C_{j}}\left(\left(s_{i}\right)_{p}-\right.$ $\left.\left(s_{j}\right)_{p}\right)$. 
Since all of the rank 2 sheaves $\mathcal{I} /\left.\mathcal{I}^{2}\right|_{C_{i}} \cong \mathcal{I} / \mathcal{I}_{i} \mathcal{I}$ are all $(0,0)$ or $(0,1)$, the group $H^{1}\left(\bigoplus_{i=1}^{n} \mathcal{I} /\left.\mathcal{I}^{2}\right|_{C_{i}}\right)$ is the trivial group and

$$
\begin{aligned}
0 \rightarrow H^{0}\left(\mathcal{I} / \mathcal{I}^{2}\right) \rightarrow H^{0}\left(\bigoplus_{i=1}^{n} \mathcal{I} /\left.\mathcal{I}^{2}\right|_{C_{i}}\right) & \\
& \rightarrow H^{0}\left(\bigoplus_{p \in C_{i} \cap C_{j}} \mathcal{I} /\left.\mathcal{I}^{2}\right|_{p}\right) \rightarrow H^{1}\left(\mathcal{I} / \mathcal{I}^{2}\right) \rightarrow 0,
\end{aligned}
$$

is the resulting cohomology exact sequence.

Now, $\mathcal{I} /\left.\mathcal{I}^{2}\right|_{p}$ is a two dimensional vector space, as it is a rank two vector bundle over a point $p$, and $\mathcal{I} /\left.\mathcal{I}^{2}\right|_{C_{i}}$ is locally free of rank two on $C_{i}$, so $H^{0}\left(\bigoplus_{i=1}^{n} \mathcal{I} /\left.\mathcal{I}^{2}\right|_{C_{i}}\right) \longrightarrow H^{0}\left(\bigoplus_{p \in C_{i} \cap C_{j}} \mathcal{I} /\left.\mathcal{I}^{2}\right|_{p}\right)$ is a surjective map, or, equivalently, $H^{1}\left(\mathcal{I} / \mathcal{I}^{2}\right)=0$. Furthermore, since $\mathcal{I} / \mathcal{I}_{i} \mathcal{I}$ decomposes as $(0,1)$ on both $C_{1}$ and $C_{n}$ and as $(0,0)$ on each of $C_{2}, \ldots, C_{n-1}, h^{0}\left(\bigoplus_{i=1}^{n} \mathcal{I} / \mathcal{I}_{i} \mathcal{I}\right)=$ $3+2(n-2)+3=2 n+2$. Also, we have $h^{0}\left(\mathcal{I} /\left.\mathcal{I}^{2}\right|_{p}\right)=2$ at each point of intersection, so $h^{0}\left(\bigoplus_{p \in C_{i} \cap C_{j}} \mathcal{I} /\left.\mathcal{I}^{2}\right|_{p}\right)=2(n-1)$ since there are $n-1$ points of intersection. In particular, then, it has been shown that

$$
h^{0}\left(\mathcal{I} / \mathcal{I}^{2}\right)=2 n+2-(2 n-2)=4
$$

and

$$
H^{1}\left(\mathcal{I} / \mathcal{I}^{2}\right)=0 .
$$

As the map on global sections, $H^{0}\left(\mathcal{I} / \mathcal{I}^{2}\right) \longrightarrow H^{0}\left(\bigoplus_{i=1}^{n} \mathcal{I} /\left.\mathcal{I}^{2}\right|_{C_{i}}\right)$ is an isomorphism away from the singular points, $H^{0}\left(\mathcal{I} / \mathcal{I}^{2}\right) \longrightarrow H^{0}\left(\mathcal{I} /\left.\mathcal{I}^{2}\right|_{C_{i}}\right)$ is surjective for each $i$. From the normalization of $C$ and the higher order neighborhoods of $C$,

$$
0 \longrightarrow \mathcal{I}^{m} / \mathcal{I}^{m+1} \longrightarrow \bigoplus_{i=1}^{n} \mathcal{I}^{m} /\left.\mathcal{I}^{m+1}\right|_{C_{i}} \longrightarrow \bigoplus_{p \in C_{i} \cap C_{j}} \mathcal{I}^{m} /\left.\mathcal{I}^{m+1}\right|_{p} \longrightarrow 0
$$

is exact, where $\mathcal{I}^{m} /\left.\mathcal{I}^{m+1}\right|_{C_{i}}=S^{m}\left(\mathcal{I} / \mathcal{I}_{i} \mathcal{I}\right)$ has $h^{1}=0$. Therefore, $H^{0}(\hat{\mathcal{I}}) \rightarrow$ $H^{0}\left(\mathcal{I} / \mathcal{I}^{2}\right)$ is surjective as well.

Recall that $\mathcal{I}_{i} / \mathcal{I}_{i}^{2}=(1,1)$ or $(0,2)$, and $\mathcal{I} / \mathcal{I}_{i} \mathcal{I}=(0,1)$ or $(0,0)$, are both generated by global sections. So, by lifting to global sections of $\mathcal{I} / \mathcal{I}^{2}$ :

Lemma 3.1. $\mathcal{I} / \mathcal{I}^{2}$ is generated by global sections.

These four global sections of $\mathcal{I} / \mathcal{I}^{2}$, lifted to global sections of $\hat{\mathcal{I}}$, define a formal cDV modification $\hat{f}: \hat{X} \rightarrow \hat{\mathbf{C}}^{4}$ for which $\hat{f}^{-1}(0)=\hat{\mathcal{I}}$, so to determine the singularity from contracting $C$, the general section of the ideal sheaf $\hat{\mathcal{I}}$ must be determined.

Proposition 3.2. The zero scheme of a general section of $\hat{\mathcal{I}}$ is a smooth surface along $C$. 
Proof. A general section of $\hat{\mathcal{I}}$ at any point of intersection $p$ is of the form $g \cdot x y+h \cdot z$ with $g, h \in \mathcal{O}_{p, \hat{X}}$ and $g$ or $h$ is a unit. Considering this as a local section of $\mathcal{I} / \mathcal{I}^{2}$, there exists a global section $s \in \mathcal{I} / \mathcal{I}^{2}$ that does not vanish at $p$, and, therefore, $h(p) \neq 0$. So, $s$ is nonsingular at $p$. The condition $h(p) \neq 0$ defines an open dense subset of $X$ on which $h$ is non-vanishing. Since $h(p) \neq 0$ at each point of intersection, and the intersection of these sets is open and dense in $X$, a general section of $\mathcal{I} / \mathcal{I}^{2}$ is nonsingular at each point of intersection. As $H^{0}(\hat{\mathcal{I}}) \rightarrow H^{0}\left(\mathcal{I} / \mathcal{I}^{2}\right)$ is surjective, lift this to a global section of $\hat{\mathcal{I}}$.

At a smooth point of $C$, a general section of $\hat{\mathcal{I}}$ is of the form $g \cdot x+h \cdot z$ with $g$ or $h$ a unit. Therefore, a general section of $\hat{\mathcal{I}}$ is smooth away from the singular points of $C$ as well.

Take a general nonzero section $s \in H^{0}\left(\mathcal{I} / \mathcal{I}^{2}\right)$. With the first map being multiplication by $s$, we have

$$
0 \longrightarrow \mathcal{O}_{C} \longrightarrow \mathcal{I} / \mathcal{I}^{2} \longrightarrow \mathcal{I}_{C, S} / \mathcal{I}_{C, S}^{2} \longrightarrow 0,
$$

where $S$ is the smooth surface defined by $s$. Restricting to $C_{i}$, then, $\mathcal{I}_{C, S} /\left.\mathcal{I}_{C, S}^{2}\right|_{C_{i}} \cong \mathcal{O}_{C_{i}}(1)$ for $i=1, n$ and $\mathcal{I}_{C, S} /\left.\mathcal{I}_{C, S}^{2}\right|_{C_{i}} \cong \mathcal{O}_{C_{i}}$ for $2 \leq i \leq n-1$. Invertible sheaves on $C$ are completely determined by their degree on each component, so it will be written $\mathcal{I}_{C, S} / \mathcal{I}_{C, S}^{2} \cong \mathcal{O}_{C}(1,0, \ldots, 0,1)$. Since $S$ is smooth, at a point of intersection $p \in C$, coordinates can be chosen so that $(s)$ is defined by $(z=0)$ and $\mathcal{I}_{C, S}=(x y)$. From the injection $\mathcal{I}_{C, S} /\left.\mathcal{I}_{C, S}^{2}\right|_{C_{i}} \hookrightarrow \mathcal{I}_{C_{i}, S} / \mathcal{I}_{C_{i}, S}^{2}$, which is an isomorphism away from the singular points of $C$, local coordinates show that the determinant map vanishes to order 1 at each point of intersection. Therefore, $\mathcal{I}_{C_{i}, S} / \mathcal{I}_{C_{i}, S}^{2} \cong \mathcal{O}_{C_{i}}(2)$ for all $1 \leq i \leq n$. We have:

Theorem 3.3. If $f: X \rightarrow Y$ is an analytic contraction map with $f(C)=q$ and $C=\bigcup_{i=1}^{n} C_{i}$ with all components having length 1 , then a general hyperplane section of $q$ has an $A_{n}$ type singularity at $q$.

\section{Contraction criteria for $\mathrm{C}$.}

In this section it will be shown how the splitting of Sequence (3) is equivalent to the existence of an infinitesimal deformation of $C$. Then, using the theory of deformations of the $A_{n}$ singularity, it will be shown that the continued splitting of related sequences determine higher order deformations of $C$.

The discussion following Sequence (3) in Section 3 shows $\mathcal{I}_{C, S} / \mathcal{I}_{C, S}^{2} \cong$ $\mathcal{O}_{C}(1,0, \ldots, 0,1)$, which is the dual of the dualizing sheaf on $C, \omega_{C}^{*}$. Sequence (3), then, will be written

$$
0 \longrightarrow \mathcal{O}_{C} \longrightarrow \mathcal{I} / \mathcal{I}^{2} \longrightarrow \omega_{C}^{*} \longrightarrow 0 \text {. }
$$


Lemma 4.1. Sequence (4) splits if and only if there exists an ideal $\mathcal{K}_{2}$ satisfying $\mathcal{I}^{2} \subset \mathcal{K}_{2} \subset \mathcal{I}, \mathcal{I} / \mathcal{K}_{2} \cong \mathcal{O}_{C}$ and $\mathcal{K}_{2} / \mathcal{I}^{2} \cong \omega_{C}^{*}$.

Proof. If this sequence splits define

$$
\mathcal{K}_{2}=\operatorname{Ker}\left(\mathcal{I} \rightarrow \mathcal{I} / \mathcal{I}^{2} \rightarrow \mathcal{O}_{C}\right) .
$$

By definition, then, $\mathcal{I}^{2} \subset \mathcal{K}_{2} \subset \mathcal{I}$ and $\mathcal{I} / \mathcal{K}_{2} \cong \mathcal{O}_{C}$. The sheaf $\mathcal{K}_{2} / \mathcal{I}^{2}$ is invertible on $C$ and restricting to each component $C_{i}$ we have

$$
0 \longrightarrow \mathcal{K}_{2} /\left.\mathcal{I}^{2}\right|_{C_{i}} \longrightarrow \mathcal{I} /\left.\mathcal{I}^{2}\right|_{C_{i}} \longrightarrow \mathcal{I} /\left.\mathcal{K}_{2}\right|_{C_{i}} \longrightarrow 0 \text {. }
$$

But, $\mathcal{I} /\left.\mathcal{K}_{2}\right|_{C_{i}} \cong \mathcal{O}_{i}$ for all $1 \leq i \leq n, \mathcal{I} /\left.\mathcal{I}^{2}\right|_{C_{i}} \cong \mathcal{O}_{i}(1) \oplus \mathcal{O}_{i}$ for $i=1, n$, and $\mathcal{I} /\left.\mathcal{I}^{2}\right|_{C_{i}} \cong \mathcal{O}_{i} \oplus \mathcal{O}_{i}$ for $2 \leq i \leq n-1$. Therefore, this sequence must split for all $1 \leq i \leq n$ and $\mathcal{K}_{2} / \mathcal{I}^{2} \cong \mathcal{O}_{C}(1,0, \ldots, 0,1) \cong \omega_{C}^{*}$.

The converse is immediate from the properties of $\mathcal{K}_{2}$ since

$$
0 \longrightarrow \mathcal{K}_{2} / \mathcal{I}^{2} \longrightarrow \mathcal{I} / \mathcal{I}^{2} \longrightarrow \mathcal{I} / \mathcal{K}_{2} \longrightarrow 0
$$

is exact.

The following proposition relates the splitting of Sequence (4), or, equivalently, the existence of the defining ideal sheaf $\mathcal{K}_{2}$, to the infinitesimal deformation of $C$.

Proposition 4.2. $C$ has an infinitesimal deformation if and only if there exists an ideal sheaf $\mathcal{K}_{2}$ satisfying $\mathcal{I}^{2} \subset \mathcal{K}_{2} \subset \mathcal{I}, \mathcal{I} / \mathcal{K}_{2} \cong \mathcal{O}_{C}$ and $\mathcal{K}_{2} / \mathcal{I}^{2} \cong$ $\omega_{C}^{*}$.

Proof. This second condition is equivalent to the splitting of the dual of Sequence (4), which is

$$
0 \longrightarrow \omega_{C} \longrightarrow \mathcal{N}_{C} \longrightarrow \mathcal{O}_{C} \longrightarrow 0,
$$

and the infinitesimal deformations are classified by $H^{0}\left(C, \mathcal{N}_{C}\right)$. Now

$$
\operatorname{Ext}_{\mathcal{O}_{C}}^{1}\left(\mathcal{O}_{C}, \omega_{C}\right) \cong H^{1}\left(C, \omega_{C}\right) \cong \mathbf{C}
$$

and the geometric genus of $C$ is 0 , so the long exact cohomology sequence can be written

$$
0 \longrightarrow H^{0}\left(\mathcal{N}_{C}\right) \longrightarrow \mathbf{C} \stackrel{\delta}{\longrightarrow} \mathbf{C} \longrightarrow H^{1}\left(\mathcal{N}_{C}\right) \longrightarrow 0,
$$

where $\delta$ is the coboundary map given by $1 \mapsto$ extension class of $\mathcal{O}_{C}$ by $\omega_{C}$. Therefore, $\delta$ is either an isomorphism or the zero map. Sequence (5) splits if and only if $\delta$ is the zero map, which is equivalent to $H^{0}\left(\mathcal{N}_{C}\right)=\mathbf{C}$.

It will now be shown that the sheaf $\mathcal{K}_{2} / \mathcal{I} \mathcal{K}_{2}$ determines the existence of higher order deformations of $C$. Calculating in local coordinates $\{x, y, z\}$ at a singular point of $C$, there are two possible forms for $\mathcal{K}_{2}$.

The invertible sheaf $\mathcal{K}_{2} / \mathcal{I}^{2}$ is a subsheaf of $\mathcal{I} / \mathcal{I}^{2}$, which is generated by $\{x y, z\}$. Therefore, $\mathcal{K}_{2} / \mathcal{I}^{2}$ is generated locally by an element of the form $f_{0} x y+f_{1} z$ with $f_{0}, f_{1} \in \mathcal{O}_{p, X}$ with either $f_{0}$ or $f_{1}$ a unit. If $f_{0}$ is a unit, 
then, dividing by $f_{0}, \mathcal{K}_{2} / \mathcal{I}^{2}$ is generated by an element of the form $x y+\lambda_{1} z$. So,

$$
\mathcal{K}_{2}=\left(x y+\lambda_{1} z\right)+\mathcal{I}^{2}=\left(x y+\lambda_{1} z, z^{2}\right) .
$$

On the other hand, if $f_{1}$ is a unit, then, dividing by $f_{1}, \mathcal{K}_{2} / \mathcal{I}^{2}$ is generated by an element of the form $g_{0} x y+z$. In this case, the analytic change of coordinates inverse to $(x, y, z) \mapsto\left(x, y, g_{0} x y+z\right)$ gives $\mathcal{K}_{2} / \mathcal{I}^{2}$ being generated by $z$, and it does not affect the description of $\mathcal{I}$ as $(x y, z)$. It can now be seen that

$$
\mathcal{K}_{2}=(z)+\mathcal{I}^{2}=\left(x^{2} y^{2}, z\right) .
$$

In either case, though, the kernel of the map $\mathcal{O}_{C} \oplus \mathcal{O}_{C} \longrightarrow \mathcal{K}_{2} / \mathcal{I} \mathcal{K}_{2}$ defined by $(f, g) \mapsto f\left(x y+\lambda_{1} z\right)+g z^{2}$ or $(f, g) \mapsto f x^{2} y^{2}+g z$ is $\mathcal{I}_{C} \oplus \mathcal{I}_{C}$. Therefore, $\mathcal{K}_{2} / \mathcal{I K}_{2}$ is locally free of rank 2 on $C$.

Furthermore, the invertible sheaf $\mathcal{I}^{2} / \mathcal{I} \mathcal{K}_{2} \cong \mathcal{I} / \mathcal{K}_{2} \otimes \mathcal{I} / \mathcal{K}_{2} \cong \mathcal{O}_{C}$, so the exact sequence

$$
0 \longrightarrow \mathcal{I}^{2} / \mathcal{I K}_{2} \longrightarrow \mathcal{K}_{2} / \mathcal{I K}_{2} \longrightarrow \mathcal{K}_{2} / \mathcal{I}^{2} \longrightarrow 0
$$

can be written

$$
0 \longrightarrow \mathcal{O}_{C} \longrightarrow \mathcal{K}_{2} / \mathcal{I} \mathcal{K}_{2} \longrightarrow \omega_{C}^{*} \longrightarrow 0,
$$

which is the same as Sequence (4) with $\mathcal{K}_{2} / \mathcal{I} \mathcal{K}_{2}$ replacing $\mathcal{I} / \mathcal{I}^{2}$. So, the process continues to determine higher order deformations of $C$.

Lemma 4.3. Let $\mathcal{K}_{m} \subset \mathcal{K}_{m-1} \subset \cdots \subset \mathcal{K}_{3} \subset \mathcal{K}_{2} \subset \mathcal{K}_{1}=\mathcal{I}$ be a sequence of ideals satisfying $\mathcal{I K}_{i-1} \subset \mathcal{K}_{i} \subset \mathcal{K}_{i-1}, \mathcal{K}_{i-1} / \mathcal{K}_{i} \cong \mathcal{O}_{C}$ and $\mathcal{K}_{i} / \mathcal{I K}_{i-1} \cong \omega_{C}^{*}$. Then:

1) In local coordinates at $p$ on $C, \mathcal{K}_{i}=\left(x y+\lambda_{1} z+\cdots \lambda_{i-1} z^{i-1}, z^{i}\right)$ for all $1 \leq i \leq m$, or $\mathcal{K}_{i}=\left(x^{i} y^{i}, z\right)$ for all $1 \leq i \leq m$.

2) $\mathcal{I K}_{i-1} / \mathcal{I} \mathcal{K}_{i} \cong \mathcal{I} / \mathcal{K}_{2} \otimes \mathcal{K}_{i-1} / \mathcal{K}_{i} \cong \mathcal{O}_{C}$ for all $1 \leq i \leq m$.

3) $\mathcal{K}_{i} / \mathcal{I} \mathcal{K}_{i}$ is locally free of rank 2 for all $1 \leq i \leq m$.

4) The sequence $0 \longrightarrow \mathcal{I K}_{i-1} / \mathcal{I K}_{i} \longrightarrow \mathcal{K}_{i} / \mathcal{I} \mathcal{K}_{i} \longrightarrow \mathcal{K}_{i} / \mathcal{I K}_{i-1} \longrightarrow 0$ is split exact for all $2 \leq i \leq m-1$.

We will now establish the relationship between the existence of the defining ideals $\mathcal{K}_{i}$ and the deformation of the curve $C$ as determined from the semi-universal deformations of $Y_{0}$ and $X_{0}$. In particular, it will be shown explicitly, from the defining equations for $X$ (see Equation (2) in Section 2.2) in the deformation theory of the $A_{n}$ singularity, that the existence of $K_{i}$ corresponds to an $i$ th order deformation of $C$. From Theorems 2.1 and 2.2 in Section 2.2, and the paragraph preceding them, it will be shown how the existence of the higher order neighborhoods of $C$, defined by the $\mathcal{K}_{i}$, coincide with certain vanishing properties of the $t^{i}$ terms of the functions $g_{i}$ (see Equation (1), Section 2.2) defining the discriminant locus of $\hat{g}: \operatorname{Spec} C[[t]] \rightarrow \operatorname{Spec} C\left[\left[t_{1}, \ldots, t_{n+1}\right]\right]$. An inductive argument on the number of components of $C$ and on the order of the terms will be utilized. 
Theorem 4.4. $C$ deforms formally in $\hat{X}$ if and only if there exists an infinite chain of subsheaves $\cdots \subset \mathcal{K}_{m+1} \subset \mathcal{K}_{m} \subset \cdots \subset \mathcal{K}_{2} \subset \mathcal{I}$ such that $\mathcal{K}_{m} / \mathcal{K}_{m+1} \cong \mathcal{O}_{C}$ and $\mathcal{K}_{m+1} / \mathcal{I} \mathcal{K}_{m} \cong \omega_{C}^{*}$, where $\omega_{C}^{*}$ is the dual of the dualizing sheaf.

Proof. The case where $C$ is a smooth rational curve has been proved by Reid in [14], the "Pagoda" construction. A brief discussion of Reid's results will be given to compare to the multiple component case.

Case 1. $\mathbf{C}=\mathbf{C}_{\mathbf{1}}$.

Using the transition functions defining $X$ in the deformation of an $A_{1}$ singularity from Section 2.2, Equation (2), and expanding the $g_{i}$ in power series form as in Equation (1), Section 2, we have

$$
\begin{aligned}
x & =u_{1}^{2} y+u_{1} \sum_{j=1}^{\infty}\left(a_{1 j}-a_{2 j}\right) t^{j} \\
t & =t \\
v_{1} & =1 / u_{1} .
\end{aligned}
$$

The curve $C$ is given by $y=t=0$ in the $\left\{u_{1}, y, t\right\}$ coordinate patch, and by $x=t=0$ in the $\left\{v_{1}, x, t\right\}$ patch. In other words, the ideal sheaf of $C$ in $X$ is $\mathcal{I}=(y, t)=(x, t)$.

Since $\mathcal{I} / \mathcal{I}^{2}$ decomposes as $(1,1)$ or $(0,2)$, there is a surjection $\mathcal{I} / \mathcal{I}^{2} \rightarrow \mathcal{O}_{C}$ if and only if $\mathcal{I} / \mathcal{I}^{2}=(0,2)$. It has been shown this is also equivalent to the existence of $\mathcal{K}_{2}$ (Lemma 4.1) and the existence of an infinitesimal deformation of $C$ (Proposition 4.2).

Lemma 4.5. There is a surjection $\mathcal{I} / \mathcal{I}^{2} \rightarrow \mathcal{O}_{C}$ if and only if $a_{11}=a_{21}$.

Proof. It suffices to show $\mathcal{I} / \mathcal{I}^{2}=(0,2)$ if and only if $a_{11}=a_{21}$. In calculating the decomposition of the conormal sheaf from the transition function, since $t^{j} \in \mathcal{I}^{2}$ for $j \geq 2$, it is only necessary to consider $x=$ $u_{1}^{2} y+u_{1}\left(a_{11}-b_{21}\right) t$. $\mathcal{I} / \mathcal{I}^{2}$ is generated locally by $\{y, t\}$ and $\{x, t\}$ in these two coordinate patches and

$$
(x, t)=\left(\begin{array}{cc}
u_{1}^{2} & \left(a_{11}-a_{21}\right) u_{1} \\
0 & 1
\end{array}\right)\left(\begin{array}{l}
y \\
t
\end{array}\right) .
$$

This matrix, in particular the entry $\left(a_{11}-a_{21}\right)$, determines $\mathcal{I} / \mathcal{I}^{2}$. That is, $\mathcal{I} / \mathcal{I}^{2}=(0,2)$ if and only if $a_{11}=a_{21}$ (see $[\mathbf{1 2}$, pp. 519-520]).

The map $\mathcal{I} / \mathcal{I}^{2} \rightarrow \mathcal{O}_{C}$, if it exists, can be calculated explicitly in coordinates to determine the ideal $\mathcal{K}_{2}$. Reid [14] shows that $\mathcal{K}_{2} / \mathcal{I}^{2} \cong \mathcal{O}_{C}(2) \cong \omega_{C}^{*}$ and $\mathcal{K}_{2}=\left(y, t^{2}\right)=\left(x, t^{2}\right)$ in local coordinates. From Sequence $(5)$, then, $\mathcal{K}_{2} / \mathcal{I K}_{2}=(1,1)$ or $(0,2)$, and the process can be continued. 
Assume that we have $K_{m} \subset \mathcal{K}_{m-1} \subset \cdots \subset \mathcal{K}_{2} \subset \mathcal{I}$ with $\mathcal{K}_{i-1} / \mathcal{K}_{i} \cong \mathcal{O}_{C}$, $\mathcal{K}_{i} / \mathcal{I} \mathcal{K}_{i-1} \cong \mathcal{O}_{C}(2)$ and $\mathcal{K}_{i} / \mathcal{I} \mathcal{K}_{i}=(0,2)$ if and only if $a_{1, i}=a_{2, i}$. Induction, with details eliminated, proves:

Lemma 4.6. There is a surjection $\mathcal{K}_{m+1} / \mathcal{I} \mathcal{K}_{m+1} \longrightarrow \mathcal{O}_{C}$ if and only if $a_{1(m+1)}=a_{2(m+1)}$.

From this inductive argument and the fact that $C$ deforms in $X$ if and only if $g_{1}(t)=g_{2}(t)$ (Theorem 2.1, Section 2.2), the following results of Reid [14] have been established:

Theorem 4.7 ([14, Reid] $). C \cong \mathbf{P}^{\mathbf{1}}$ deforms in $X$ if and only if there exists an infinite chain $\cdots \subset \mathcal{K}_{m+1} \subset \mathcal{K}_{m} \subset \cdots \mathcal{K}_{2} \subset \mathcal{I}$ satisfying $\mathcal{K}_{m} / \mathcal{K}_{m+1} \cong \mathcal{O}_{C}$ and $\mathcal{K}_{m+1} / \mathcal{I K}_{m} \cong \mathcal{O}_{C}(2)$.

Furthermore, if for some $m, \mathcal{K}_{m} / \mathcal{I} \mathcal{K}_{m}=(1,1)$, then $g_{1}(t) \neq g_{2}(t)$. Therefore:

Theorem $4.8([\mathbf{1 4}$, Reid $]) . C \cong \mathbf{P}^{\mathbf{1}}$ contracts if and only if the chain $\cdots \subset$ $\mathcal{K}_{m} \subset \cdots \subset \mathcal{K}_{2} \subset \mathcal{I}$ terminates.

Remark 4.9. Reid, in [14], showed not only that $C$ contracts or deforms in this formal structure, but also that there is actually an analytic deformation or contraction of $C$.

To extend to multiple components, it will first be shown for two components and it is without loss of generality (and to avoid sub-subscripts) that the first two components are used.

Case 2. $C=C_{1} \cup C_{2}$.

From the description of $X$ by transition functions in Equation (2), if $i=1$ and $n=2$, then $X$ is defined by the transition functions

$$
\begin{array}{rlrl}
x & =u_{1}^{2} v_{2}+u_{1}\left(g_{1}(t)-g_{2}(t)\right) & u_{2} & =1 / v_{2} \\
v_{1} & =1 / u_{1} & y & =v_{2}^{2} u_{1}+v_{2}\left(g_{3}(t)-g_{2}(t)\right) \\
t & =t & t & t
\end{array}
$$

where $\mathcal{I}=\left(u_{1} v_{2}, t\right)=(x, t)=(y, t), \mathcal{I}_{1}=\left(v_{2}, t\right)=(x, t)$ and $\mathcal{I}_{2}=\left(u_{1}, t\right)=$ $(y, t)$ in the coordinate patches $\left(u_{1}, v_{2}, t\right),\left(x, v_{1}, t\right)$ and $\left(u_{2}, y, t\right)$.

Lemma 4.10. There is a surjection $\mathcal{I} / \mathcal{I}^{2} \longrightarrow \mathcal{O}_{C}$ if and only if $a_{11}=a_{31}$.

Proof. Assume that there is a surjection $\mathcal{I} / \mathcal{I}^{2} \rightarrow \mathcal{O}_{C}$. Defining this in local coordinates on the patch $\left(u_{1}, v_{2}, t\right)$ containing the point of intersection, let $u_{1} v_{2} \mapsto h_{1}\left(u_{1}, v_{2}\right)$ and $t \mapsto h_{2}\left(u_{1}, v_{2}\right)$ where the $h_{i}$ are holomorphic functions in $u_{1}$ and $v_{2}$. Since $t^{2} \in \mathcal{I}^{2}$ in each patch, it suffices to assume $g_{i}(t)=a_{i 1} t$ for $1 \leq i \leq 3$. Then, in the remaining coordinate patches, $\mathcal{I} / \mathcal{I}^{2} \rightarrow \mathcal{O}_{C}$ is given by

$$
\begin{array}{rlrl}
x & \mapsto u_{1} h_{1}+u_{1}\left(a_{11}-a_{21}\right) h_{2} & y & \mapsto v_{2} h_{1}+v_{2}\left(a_{31}-a_{21}\right) h_{2} \\
t & \mapsto h_{2} & t & \mapsto h_{2} .
\end{array}
$$


The images of the generators $\{x, t\}$ and $\{y, t\}$ of $\mathcal{I} / \mathcal{I}^{2}$ must be holomorphic in the coordinate patches $\left(x, v_{1}, t\right)$ and $\left(u_{2}, y, t\right)$ respectively. In particular, $h_{2}$ must be holomorphic in the coordinate $v_{1}=1 / u_{1}$ and in $u_{2}=1 / v_{2}$. This can only be possible if $h_{2}$ is a constant function. Let $h_{2}=c$ where $c \in \mathbf{C}$ and $c \neq 0$ for a nontrivial map.

Viewing $h_{1}$ as a power series in $u_{1}$ and $v_{2}$, the image of $x, u_{1} h_{1}+u_{1}\left(a_{11}-\right.$ $\left.a_{21}\right) c$, can only be holomorphic in $v_{1}$ if it is the zero function. Therefore, $h_{1}=\left(a_{21}-a_{11}\right) c$. The surjection can exist, then, only if in the coordinates $\{y, t\}$,

$$
\begin{aligned}
y & \mapsto v_{2}\left(a_{21}-a_{11}\right) c+v_{2}\left(a_{31}-a_{21}\right) c \\
t & \mapsto c
\end{aligned}
$$

with the image of $y$ holomorphic in $u_{2}=1 / v_{2}$. Again, this is only possible if it is the zero function, which is equivalent to $\left(a_{21}-a_{11}\right)=\left(a_{21}-a_{31}\right)$ or $a_{11}=a_{31}$.

Furthermore, assuming $c=1$ (since $c \neq 0$ ) and letting $\lambda_{1}=a_{11}-a_{21}=$ $a_{31}-a_{21}$, the surjection is defined on the generators by the equations

$$
\begin{array}{lllllll}
u_{1} v_{2} & \mapsto-\lambda_{1} & x & \mapsto & 0 & y & \mapsto \\
t & \mapsto 1 & t & \mapsto & 1 & t & \mapsto
\end{array}
$$

Conversely, if $a_{11}=a_{31}$ define $\mathcal{I} / \mathcal{I}^{2} \longrightarrow \mathcal{O}_{C}$ by the above equations.

The subsheaf $\mathcal{K}_{2}$ of $\mathcal{I}$ satisfying the conditions of Lemma 4.1 can also be calculated explicitly using this local description. By definition, $\mathcal{K}_{2}=$ $\operatorname{Ker}\left(\mathcal{I} \rightarrow \mathcal{I} / \mathcal{I}^{2} \rightarrow \mathcal{O}_{C}\right)$, so $t^{2} \in \mathcal{I}^{2}$ and $\left\{u_{1} v_{2}+\lambda_{1} t, t^{2}\right\}$ generate $\mathcal{K}_{2}$ in the $\left(u_{1}, v_{2}, t\right)$ patch. Similarly, from the equations of the map $\mathcal{I} / \mathcal{I}^{2} \rightarrow \mathcal{O}_{C}$ above $\left\{x, t^{2}\right\}$ and $\left\{y, t^{2}\right\}$ generate $\mathcal{K}_{2}$ in the $\left(x, v_{1}, t\right)$ and $\left(u_{2}, y, t\right)$ patches, respectively. Notice that this local description of $\mathcal{K}_{2}$ is equivalent to one of the forms that was determined without the use of the equations from the deformations of the $A_{n}$ singularity (see Lemma 4.3, Part 1). To complete the induction:

Lemma 4.11. There is a surjection $\mathcal{K}_{m+1} / \mathcal{I} \mathcal{K}_{m+1} \rightarrow \mathcal{O}_{C}$ if and only if $a_{1(m+1)}=a_{3(m+1)}$.

Proof. Assume there is a surjection $\mathcal{K}_{k} / \mathcal{I} \mathcal{K}_{k} \rightarrow \mathcal{O}_{C}$ if and only if $a_{1 k}=a_{3 k}$ for all $k \leq m$.

To extend this to $m+1$, notice that $\mathcal{K}_{m+1} / \mathcal{I} \mathcal{K}_{m+1}$ is generated by $\left\{u_{1} v_{2}+\right.$ $\left.\lambda_{1} t+\cdots+\lambda_{m} t^{m}, t^{m+1}\right\}$ at the point of intersection, where $\lambda_{i}=a_{1 i}-$ $a_{2 i}=a_{3 i}-a_{2 i}$, and by $\left\{x, t^{m+1}\right\}$ and $\left\{y, t^{m+1}\right\}$ on the other coordinate patches. Since $t^{m+2} \in \mathcal{I K}_{m+1}$ in each patch, to calculate the surjection $\mathcal{K}_{m+1} / \mathcal{I} \mathcal{K}_{m+1} \rightarrow \mathcal{O}_{C}$, it suffices to consider $g_{i}(t)=\sum_{j=1}^{m+1} a_{i j} t^{j}$ for $i=1,2,3$. 
Defining this map on generators in the coordinates $\left(u_{1}, v_{2}, t\right)$, let

$$
\begin{aligned}
u_{1} v_{2}+\lambda_{1} t \cdots+\lambda_{m} t^{m} & \mapsto h_{1}\left(u_{1}, v_{2}\right) \\
t^{m+1} & \mapsto h_{2}\left(u_{1}, v_{2}\right)
\end{aligned}
$$

where $h_{1}$ and $h_{2}$ are holomorphic in $u_{1}$ and $v_{2}$. In the other patches we also have the generator $t^{m+1}$ mapping to the function $h_{2}$. The exact reasoning from Lemma 4.10 shows that $h_{2}$ must be the constant function, and it can be assumed to be the constant 1 . So, the surjection on the remaining generators is given by

$$
\begin{array}{llll}
x & \mapsto u_{1}\left(h_{1}+a_{1(m+1)}-a_{2(m+1)}\right) & y & \mapsto v_{1}\left(h_{1}+a_{3(m+1)}-a_{2(m+1)}\right) \\
t^{m+1} & \mapsto 1 & t^{m+1} & \mapsto 1 .
\end{array}
$$

As for the surjection from $\mathcal{I} / \mathcal{I}^{2}$ done previously, we can conclude that $h_{1}=$ $a_{2(m+1)}-a_{1(m+1)}=a_{2(m+1)}-a_{3(m+1)}$, and this surjection can occur if and only if $a_{1(m+1)}=a_{3(m+1)}$.

Therefore, for the case where $C=C_{1} \cup C_{2}, C$ deforms if and only if there is an infinite chain $\cdots \subset \mathcal{K}_{m} \subset \cdots \mathcal{K}_{2} \subset \mathcal{I}$ with $\mathcal{K}_{m} / \mathcal{K}_{m+1} \cong \mathcal{O}_{C}$ and $\mathcal{K}_{m+1} / \mathcal{I} \mathcal{K}_{m} \cong \omega_{C}^{*}$.

Induction on more components of $C$ is immediate as it is necessary to show that the surjection is well-defined where $C_{m+1}$ intersects $\cup_{i=1}^{m} C_{m}$, which is just the point $p=C_{m} \cap C_{m+1}$. This completes the proof of Theorem 4.4.

Theorem 4.12. A formal $c A_{n}$ contraction of $C$ exists if and only if there is no infinite chain of subsheaves $\cdots \subset \mathcal{K}_{m+1} \subset \mathcal{K}_{m} \subset \cdots \mathcal{K}_{2} \subset \mathcal{I}_{D}$ satisfying $\mathcal{K}_{m} / \mathcal{K}_{m+1} \cong \mathcal{O}_{D}$ and $\mathcal{K}_{m+1} / \mathcal{I} \mathcal{K}_{m} \cong \omega_{D}^{*}$ for any $D=\bigcup_{j=i}^{k} C_{j}(1 \leq i \leq k \leq$ $n)$, where $\mathcal{I}_{D}$ is the ideal sheaf of $D$ in $X$.

Proof. For every $i$ and $k$ we can conclude from the proof of Theorem 4.4 that $g_{i} \neq g_{k+1}$. Therefore, the curve $D$ is not contained in the discriminant locus, which is equivalent to the induced formal map, SpecC $[[t]] \rightarrow$ Spec $\mathbf{C}^{\mathbf{n}}\left[\left[t_{1}, \ldots, t_{n}\right]\right]$ not factoring through the discriminant locus in $\operatorname{Spec}^{\mathbf{n}}\left[\left[t_{1}, \ldots, t_{n}\right]\right]$.

Notice from this theorem, it can be concluded that even if every component of $C$ can be contracted, this is not enough to ensure that $C$ contracts.

Example 4.13. Using the description of $X$ by transition functions in Equation (2) of Section 2.2, with $C=C_{1} \cup C_{2}$, let $g_{1}(t)=2 t, g_{2}(t)=t$ and $g_{3}(t)=2 t$. Since $g_{1}(t)=g_{3}(t)$, the curve $C$ deforms in $X$ and so is not contractible. However, since $g_{1}(t) \neq g_{2}(t), C_{1}$ can be contracted, and since $g_{2}(t) \neq g_{3}(t), C_{2}$ can also be contracted. In fact, $\mathcal{I}_{1} / \mathcal{I}_{1}^{2}=(1,1)$ and $\mathcal{I}_{2} / \mathcal{I}_{2}{ }^{2}=(1,1)$ (see $\left.[\mathbf{1 0}]\right)$. The conormal sheaves of each component being ample implies that $C_{1}$ and $C_{2}$ can each be contracted separately. 
Acknowledgement. This article is part of my $\mathrm{Ph} . \mathrm{D}$. dissertation supervised under Professor Sheldon Katz. I greatly appreciate his patience and many helpful suggestions. I also want to thank the referee for helping simplify the methods used in obtaining the results in Section 3.

\section{References}

[1] M. Artin, Algebraization of formal moduli, I, in 'Global Analysis', Papers in Honor of K. Kodaira, University of Tokyo Press, Princeton University Press, 1969, 21-71, MR 41 \#5369, Zbl 0205.50402.

[2] _ Algebraization of formal moduli: II. Existence of modifications, Ann. of Math., 91 (1970), 88-135, MR 41 \#5370, Zbl 0177.49003.

[3] H. Clemens, J. Kollár and S. Mori, Higher dimensional complex geometry, Astérisque, 166 (1988), MR 90j:14046, Zbl 0689.14016.

[4] H. Grauert, Über Modifikationen und exzeptionnelle analytische Mengen, Math. Ann., 146 (1962), 331-368, MR 25 \#583, Zbl 0178.42702.

[5] R. Hartshorne, Ample vector bundles, Publ. Math. IHES, 29 (1966), 63-94, MR 33 \#1313, Zbl 0173.49003.

[6] J. Jiménez, Contraction of nonsingular curves, Duke Math. J., 65(2) (1992), 313332, MR 93e:14019, Zbl 0781.3202.

[7] A. Kas, On the resolution of certain holomorphic mappings, in 'Global Analysis', Papers in honor of K. Kodaira (D.C. Spencer and S. Iyanaga, eds.), University of Tokyo Press, Tokyo, and Princeton University Press, Princeton, 1969, 289-294, MR 41 \#2047, Zbl 0192.57902.

[8] S. Katz and D. Morrison, Gorenstein threefold singularities with small resolutions via invariant theory for Weyl groups, J. Alg. Geom., 1 (1992), 449-530, MR 93b:14030, Zbl 0788.14036.

[9] Y. Kawamata, General hyperplane sections of nonsingular flops in dimension 3, Math. Res. Lett., 1(1) (1994), 49-52, MR 95d:14021, Zbl 0834.3200.

[10] H.B. Laufer, On $\mathbf{C P}^{1}$ as exceptional set, Ann. of Math. Stud., 100, Princeton University Press, Princeton, 1981, 261-275, MR 82m:32012, Zbl 0523.32007.

[11] S. Mori, Flip theorem and the existence of minimal models for 3-folds, J. Amer. Math. Soc., 1 (1988), 117-253, MR 89a:14048, Zbl 0649.14023.

[12] N. Nakayama, On smooth exceptional curves in threefolds, J. Fac. Sci. Univ. Tokyo, Tokyo, 37 (1990), 511-525, MR 92e:14035, Zbl 0739.14027.

[13] H. Pinkham, Factorization of birational maps in dimension 3, Proc. Symp. Pure Math., 40, Part 2, Amer. Math. Soc., Providence, 1983, 343-371, MR 85g:14015, Zbl 0544.14005.

[14] M. Reid, Minimal models of canonical threefolds, Algebraic varieties and analytic varieties, Adv. Studies in Pure Math., 1 (1983), 131-180, MR 86a:14010, Zbl 0558.14028.

[15] G.N. Tyurina, Resolution of singularities of flat deformations of rational double points, Functional Anal. Appl., 4 (1970), 324-356, Zbl 0221.32008.

[16] B. van der Waerden, Zur algebraischen geometrie VI: Algebraische korrespondenzen und rationale abbildungen, Math. Ann., 110 (1934), 134-160, Zbl 0009.2260. 
Received September 9, 2002.

Department of Mathematics

Saginaw Valley State University

University Center, MI 48710

E-mail address: zerger@svsu.edu 